Meta

Journal des traducteurs

Translators' Journal

\title{
Avant-propos
}

\section{Charlotte Melançon}

Volume 45, numéro 1, avril 2000

La traduction littéraire au Canada

Literary Translation in Canada

URI : https://id.erudit.org/iderudit/003617ar

DOI : https://doi.org/10.7202/003617ar

Aller au sommaire du numéro

Éditeur(s)

Les Presses de l'Université de Montréal

ISSN

0026-0452 (imprimé)

1492-1421 (numérique)

Découvrir la revue

Citer ce document

Melançon, C. (2000). Avant-propos. Meta, 45(1), 80-89.

https://doi.org/10.7202/003617ar

Ce document est protégé par la loi sur le droit d'auteur. L'utilisation des services d'Érudit (y compris la reproduction) est assujettie à sa politique d'utilisation que vous pouvez consulter en ligne.

https://apropos.erudit.org/fr/usagers/politique-dutilisation/
Cet article est diffusé et préservé par Érudit.

Érudit est un consortium interuniversitaire sans but lucratif composé de l’Université de Montréal, l'Université Laval et l'Université du Québec à Montréal. Il a pour mission la promotion et la valorisation de la recherche. https://www.erudit.org/fr/ 


\section{AVANT-PROPOS}

Ce numéro de Meta souligne le vingt-cinquième anniversaire de l'Association des traducteurs et traductrices littéraires du Canada (ATTLC). La pratique de la traduction littéraire a certes une longue histoire au Canada, dont une exposition à la Bibliothèque nationale à Ottawa témoignera en septembre 2000 et dont l'article de Judith Woodsworth rappelle ici-même un aspect. Cet anniversaire marque néanmoins une étape importante dans la reconnaissance de la traduction littéraire en tant qu'élément fondamental de la vie culturelle dans un pays bilingue.

Vingt-cinq années, c'est peu en un sens, mais beaucoup en un autre. Celles-ci ont été bien remplies: promotion du caractère spécifique de la traduction littéraire, expansion de l'ATTLC dans toutes les parties d'un pays immense et très divers, établissement de relations avec les autres associations de traducteurs, tant au niveau provincial qu'aux niveaux national et international, efforts pour établir un contrattype avec les éditeurs et pour promouvoir les intérêts matériels et moraux des traducteurs, reconnaissance du texte de la traduction comme ouvre à part entière inscrite dans la Loi canadienne sur le droit d'auteur, création du prix John-Glassco attribué à une première traduction afin d'assurer la relève. Patricia Claxton, qui fut la première présidente de l'Association, relate ses débuts difficiles. Si l'ATTLC a reçu des écrivains canadiens et de leurs associations ainsi que de divers groupes de traducteurs européens et américains un appui moral inconditionnel, le Conseil des arts du Canada a joué un rôle déterminant en lui accordant dès le début une aide financière et un soutien institutionnel indispensables. On se rappellera que les prix littéraires du Gouverneur général, que le Conseil accorde annuellement, comptaient depuis longtemps, avant même la fondation de l'ATTLC, deux prix attribués aux traducteurs littéraires, dans chacune des langues officielles du Canada, au même titre qu'aux romanciers ou aux poètes. L'histoire de l'ATTLC semble donc, après coup, se résumer facilement, mais elle a connu, comme le rappelle sa première présidente, des hauts et des bas et, est-il besoin de le rappeler, elle doit encore faire face à des difficultés. L'essentiel reste que le pari fait par un petit groupe d'une dizaine de traducteurs en 1975 a été tenu: l'ATTLC compte maintenant cent cinquante membres dans l'ensemble du pays et elle appartient pleinement à l'institution littéraire canadienne.

Ce développement et cette reconnaissance institutionnelle avaient été longuement préparés par une pratique de la traduction littéraire au Canada qui reste méconnue. Judith Woodsworth en rappelle un chapitre dans l'article qu'elle consacre à Watson Kirkconnell, poète, critique, professeur et traducteur prolifique. Cité brièvement dans la Canadian Encyclopedia sous la rubrique «Ethnic Literature», Kirkconnell est pourtant l'auteur, entre autres, d'une grande anthologie intitulée European Elegies (1928), qui rassemble une centaine de poèmes traduits de cinquante langues. J. Woodsworth retrace l'histoire de ce traducteur hors du commun, analyse sa conception de la traduction poétique et la réception de son œuvre; il est probable, dit-elle 
en conclusion, que la politique officielle du bilinguisme au Canada a occulté en partie l'apport exceptionnel de ce traducteur polyglotte. Si la traduction littéraire entre les deux langues officielles du pays a connu un développement remarquable au Canada depuis un quart de siècle, la traduction d'autres langues reste, en effet, marginale, faute de soutien du Conseil des arts et à cause de la timidité des éditeurs canadiens en ces matières, trop prompts à céder le terrain à leurs homologues britanniques et américains, français, suisses et belges. Il n'y a pas si longtemps, toutefois, même la traduction entre les deux langues officielles du pays ne devait pas vaincre moins de résistances. L'article de Patricia Godbout rappelle opportunément, à cet égard, le débat politico-littéraire qu'ont suscité les premières traductions d'Anne Hébert ou de Saint-Denys Garneau par Frank Scott ou John Glassco, et la rareté des traductions dont témoignait la Bibliographie des livres canadiens traduits de l'anglais au français et du français à l'anglais (1975) de Philip Stratford.

À cette mise en contexte historique succèdent des études de cas sur les problèmes de traduction du roman et de la poésie, ou sur des questions plus générales. C'est l'espace, ouvert et diversifié, de la traduction littéraire au Canada qui s'y donne à lire. D’autres choix auraient été possibles. Le numéro que nous proposons trouve son unité dans un parti qui équivaut à une prise de position sur la traduction. Si celle-ci, comme toute autre activité humaine, peut devenir objet de théorie, elle reste avant tout une pratique. Ce n'est pas sans raison que tous les collaborateurs de cet ensemble, membres de l'ATTLC, sont des traducteurs qui se sont mesurés à la difficulté concrète de traduire, c'est-à-dire de frayer un passage entre deux langues et de trouver ou de se résigner à un équivalent dont ils savent, les premiers, le caractère tout approximatif. "Traduire, c'est trahir», comme le veut un adage passé en cliché. Et pourtant, on traduit, parce que cette trahison est aussi un don et qu'entre l'auteur et le lecteur, lorsque s'oppose l'obstacle d'un code indéchiffrable, un passeur intervient, qui le surmonte tant bien que mal. La traduction est un art, qui appelle, toujours recommencée, sa défense et illustration. Celle-ci, du point de vue des praticiens, ne peut se faire que sur exemples, d'où ces études, partielles, partiales et, parfois, passionnées.

Betty Bednarski déploie un réseau d'échanges linguistiques complexes entre l'anglais et le français dans le roman de Jacques Ferron La nuit et dans sa version revue et amplifiée sous le titre Les confitures de coings: une activité traductrice complexe travaille le texte original de ce roman, où l'on trouve de nombreuses références à des poètes anglophones, à Samuel Butler en particulier, dont Ferron retraduit un poème - A Psalm of Montreal - à partir d'une traduction française de Valery Larbaud; la bipolarité linguistique canadienne s'y trouve refondue et enrichie par la culture universelle, ce qui propose au traducteur de passionnantes ambiguïtés. Louis Jolicœur analyse la dynamique essentielle du travail de traducteur/lecteur/interprète, tissée tout aussi bien d'ambiguïtés structurelles et immédiates, d'ambiguïtés nécessaires, néanmoins, au passage entre les langues; il compare à titre d'exemples plusieurs extraits du roman de Juan Carlos Onetti, Los adioses, dans leurs versions française, anglaise et italienne. Luise von Flotow, pour sa part, se saisit de la question des littératures dites minoritaires ou marginales en analysant les difficultés particulières auxquelles a dû faire face le traducteur anglophone de l'œuvre de l'écrivaine d'origine turque Emine Sevgi Özdamar, qui remportait récemment, malgré son broken German, le prestigieux prix Ingeborg-Bachmann. 
En déchiffrant dans le dernier roman de Gail Scott, My Paris, tout un réseau de références à des langues diverses, à Gertrude Stein, à Walter Benjamin, et en décrivant dans le renga bilingue de Jacques Brault et d'E. D. Blodgett, Transfiguration, l'établissement de passerelles entre deux traditions poétiques, Sherry Simon met en relief le fait que la traduction s'est faite petit à petit espace de rencontres dans les œuvres récentes de plusieurs auteurs canadiens, romanciers ou poètes, au point qu'il n'y a peut-être plus lieu de parler de frontières linguistiques à franchir, mais d'un espace commun à habiter. L'inscription de l'œuvre dans un lieu géographique et culturel pose au traducteur le problème peut-être insoluble de la transposition dans un nouveau contexte de lecture; Charlotte Melançon l'aborde dans un ensemble de traductions françaises — faudrait-il dire «hexagonales» — de la poésie d'Emily Dickinson. Nicole Côté, quant à elle, met en lumière les défis de traduction que posent les œuvres postmodernes en transgressant les frontières traditionnelles des genres littéraires; elle analyse le recueil de poèmes de Jane Urquhart, The Little Flowers of Madame de Montespan, œuvre protéiforme, tant par la diversité des genres auxquels elle emprunte que par celle des lieux, des époques et des voix qui y sont confrontées; ouvrant son atelier, la traductrice explique ici les partis qu'elle adopte.

Traductrice des Mémoires (1866) d'Aubert de Gaspé, Jane Brierley aborde la question d'un «je» mi-fictif et mi-réel, dans le récit autobiographique, en considérant, d'un point de vue sémantique, diverses traductions de La Vie de Henry Brulard de Stendhal. Agnes Whitfield analyse l'importance de la syntaxe - parent pauvre, semble-t-il, de la traductologie — dans la prose d'essai; par trois exemples, elle montre à quel point l'ordre des mots est essentiel au rendu du mouvement et du style, de cet impondérable qu'est le ton du texte, mais également à la cohérence générale de l'essai, autrement dit à la rigueur du discours. Spécialiste du doublage cinématographique, Robert Paquin met les cartes sur table et analyse le conflit qui oppose actuellement la France et le Canada (et le Québec francophone) sur cette question : pourquoi seraitil préférable de traduire le slang américain en argot parisien plutôt qu'en québécois? La réponse tient sans doute au monopole culturel de la France.

Bibliothécaire de profession autant que traductrice de métier, Beatriz Zeller analyse la situation des traducteurs dans le contexte de la bibliothéconomie. D'après les règles de catalogage actuellement en usage en Amérique du Nord, les traducteurs, contrairement aux éditeurs et aux compilateurs, n'ont pas droit à une main entry. Sur la base d'exemples tirés de la littérature des deux Amériques, B. Zeller montre à quel point l'idée qu'on a du traducteur reste sommaire.

Intermédiaire entre l'auteur et le lecteur, passeur entre les langues et les cultures, errant aux frontières et aux confins, le traducteur n'est pas qu'un technicien bien qu'il sache tout ce qu'il entre de technique dans le texte le plus inspiré, parce qu'il s'est mesuré concrètement, difficilement, dans le maquis des dictionnaires et des grammaires, aux problèmes techniques qui séparent un texte de sa transformation en une autre langue. Il est aussi, et peut-être d'abord, un témoin. Poèmes et romans, en toutes langues, s'efforcent, tant bien que mal, de dire l'indicible dans des langues qui élèvent des frontières, des murs de la honte, de l'exclusion et du mensonge, autant qu'elles créent un espace de civilité. Aussi ce numéro se clôt-il sur deux contributions qui rappellent que le traducteur est aussi — qu'il est d'abord - un citoyen et que les textes qu'il réécrit pour de nouveaux lecteurs ne sont pas neutres. Jean Antonin Billard a traduit sept poèmes du montréalais Irving Layton, grand poète, auteur 
d'une œuvre de premier plan non seulement par ses qualités littéraires, mais par le discours de souffrance infinie qu'elle accueille et auquel elle donne une voix: "l'intraduisible obscénité de la Shoah». Amela Simic, traductrice d'origine yougoslave, immigrée depuis peu au Canada, signe le dernier texte. Elle dit l'horreur de la guerre et le changement que son atrocité a opéré en elle. Pourquoi, tout à coup, les mots n'ont-ils plus le même sens?

Charlotte Melançon 